\title{
Blue/violet laser inactivates methicillin-resistant Staphylococcus aureus by altering its transmembrane potential
}

Gabriel Biener $^{\mathrm{a}, 1}$, Daniela S. Masson-Meyers ${ }^{\mathrm{a}, \mathrm{b}, 1}$, Violet V. Bumah ${ }^{\mathrm{a}, \mathrm{b}}$, Grant Hussey ${ }^{\mathrm{a}}$, Michael R. Stoneman ${ }^{\text {a }}$, Chukuka S. Enwemeka ${ }^{\text {b }}$, Valerica Raicu ${ }^{\text {a,c* }}$

${ }^{a}$ Department of Physics, University of Wisconsin-Milwaukee, Milwaukee, Wisconsin, USA

${ }^{\mathrm{b}}$ College of Health Sciences, University of Wisconsin-Milwaukee, Milwaukee, Wisconsin, USA

${ }^{c}$ Department of Biological Sciences, University of Wisconsin-Milwaukee, Milwaukee, Wisconsin, USA

a Department of Physics, University of Wisconsin-Milwaukee. 3135 N. Maryland Ave, Milwaukee, WI 53211, USA. Gabriel Biener: biener@uwm.edu; Grant Hussey: granthussey@gmail.com; Michael R. Stoneman: stonema2@uwm.edu; Valerica Raicu: vraicu@uwm.edu

${ }^{b}$ College of Health Sciences, University of Wisconsin-Milwaukee. 2400 E. Hartford Ave, Milwaukee, WI 53211, USA. Daniela S. Masson-Meyers: dsmasson@gmail.com; Violet V. Bumah: bumah_violet@yahoo.com; Chukuka S. Enwemeka: enwemeka@gmail.com

${ }^{c}$ Department of Biological Sciences, University of Wisconsin-Milwaukee. 3209 N Maryland Ave, Milwaukee, WI 53211, USA

\section{${ }^{1}$ G.B and D.S.M have contributed equally to this work.}

\section{*Corresponding author:}

Valerica Raicu, $\mathrm{PhD}$

Professor, Physics Department

Adjunct Professor, Department of Biological Sciences

Director, UWM-Small Businesses Collaboratory

University of Wisconsin-Milwaukee

3135 N. Maryland Ave, WI 53211, USA

Phone: (+1) 414-229-4969

E-mail:vraicu@uwm.edu 


\section{ABSTRACT}

The resistance of methicillin-resistant Staphylococcus aureus to antibiotics presents serious clinical problems that prompted the need for finding alternative or combination therapies. One such therapies is irradiation with blue light. To determine the alterations in metabolic processes implicated in the observed antimicrobial effects of blue light, we investigated the changes in membrane potential and the presence of free-radical-producing photo-acceptor molecules. Bacterial cultures irradiated with one or two doses of $405 \mathrm{~nm}$ laser light (each consisting of $121 \mathrm{~J} / \mathrm{cm}^{2}$ ) were imaged with spectrally resolved laser-scanning microscopes to detect endogenous fluorescent species as well as the voltage sensitive dye 3,3'Diethyloxacarbocyanine iodide. The endogenous fluorescence indicated the presence of photosensitizers (i.e., porphyrins, NADH, FAD) in the cells, while the exogenous signal allowed us to monitor rapid changes in transmembrane potential following treatment with light. The changes were drastic within the first 5 minutes after irradiation with the first dose and continued slowly after the second irradiation. These results suggest that the early antimicrobial activity of blue light results from alteration of membrane integrity with a consequent decrease in membrane polarization and rapid alteration of vital cellular functions. The observation of an early antimicrobial activity of light is very encouraging, as it suggests that treatment does not necessarily have to be administered over a long period of time.

Keywords: blue light; fluorescence microscopy; methicillin-resistant Staphylococcus aureus; multispectral imaging; porphyrin.

Abbreviations: MRSA, methicillin-resistant Staphylococcus aureus; MSSA, methicillinsensitive Staphylococcus aureus; ROS, reactive oxygen species; DMSO, Dimethyl sulfoxide; TM, transmembrane; OEM, original equipment manufacturer; $\mathrm{CW}$, continuous wave; $\mathrm{CFU}$, colony-forming unit; NA, numerical aperture; SL, scan lens; TL, tube lens; GS, galvanometric mirrors; EMCCD, Electron-multiplying charge coupled device; BLUF, blue-light sensing using Flavin; PYP, photoactive yellow protein; DBS, directional back scatter detector; ALA, aminolevulinic acid 


\section{Introduction}

The effectiveness of antibiotic treatment against methicillin-resistant Staphylococcus aureus (MRSA) has substantially decreased due to the development of resistant strains [1, 2]. In fact, reports indicate that death due to MRSA infection exceeds that due to HIV/AIDS [3]. The need to address this problem has prompted the pursuit of alternative therapies. Documented alternative therapies include a combination of honey and antibiotics [4], antibacterial clay [5], hyperbaric oxygen therapy [6,7] and irradiation with blue light [8-17]. Examples of conditions or infections which were successfully treated with blue light include acne vulgaris [18], eradication of Propionibacterium acnes [19], as well as different Gram-negative and Grampositive bacteria (including MRSA) upon pre-treatment with $\delta$-aminolevulinic acid (ALA) to induce prior production of sensitizers, i.e., porphyrins [20, 21].

The use of blue/violet light with no additional production of sensitizers was proposed after experiments proved to be successful in eradicating two strains of MRSA upon irradiation with $405 \mathrm{~nm}$ and $470 \mathrm{~nm}$ light [8, 9]. Since then, several reports have shown that $405 \mathrm{~nm}, 415$ $\mathrm{nm}$ and $470 \mathrm{~nm}$ blue light suppress the growth of $S$. aureus [both MRSA and methicillinsensitive S. aureus (MSSA)], Escherichia coli and other bacterial pathogens [8, 9, 12, 22-24].

Mechanisms of the phototoxicity of blue/violet light proposed in the literature center around the generation of reactive oxygen species (ROS) in mammalian and bacterial cells following the absorption of light by photoacceptor molecules including porphyrins and flavins $[10,23]$. These endogenous photosensitizers exhibit absorption spectra with peaks in the blue region of the spectrum and emissions in the green through the red region of the spectrum.

It is known that absorption of light brings porphyrin molecules into an excited triplet state, which makes it possible for a non-radiative transfer of energy [25] to the chemically stable molecular oxygen (which is also in a triplet ground state) to occur [26, 27]. The excited oxygen molecule dissociates into two highly reactive atomic species that may interact with macromolecules and damage the integrity of the cell. However, specific physiological alterations induced by the reactive species generated by light as well as the time-scale of the eradication process remain poorly understood. Such questions are relevant not only from a fundamental science standpoint, but also because their responses are likely to suggest ways to optimize the phototherapeutic protocols, in terms of total dose, treatment response time, repetition time, etc.

Studies have attempted to investigate some physiological changes/down stream effects of blue light irradiation; for example, many sequenced microbial genomes during the last few years 
have added blue-light sensing using flavin (BLUF) to the family of blue light receptors, which have highlighted the intriguing wealth of blue light sensing molecules in prokaryotes [28, 29]. In addition, functional analysis of photoactive yellow protein (PYP) in the heterotrophic deep sea bacterium Idiomarina loihiensis and cyanobacteria revealed that light suppressed biofilm formation [30, 31]. However, studies determining physiological changes are lacking.

Using a battery of spectrally resolved fluorescence techniques, both existing $[32,33]$ and newly developed for this study, we attempted to elucidate some of the questions mentioned above by detecting fluorescence from endogenous and exogenous fluorescent species in MRSA before and after irradiation with $405 \mathrm{~nm}$ laser light. After confirming the presence of fluorescence from endogenous photosensitizers (i.e., porphyrins) within the cells, we turned our attention to monitoring the changes in spectral properties of a voltage sensitive dye, 3,3'diethyloxacarbocyanine iodide $\left(\mathrm{DiOC}_{2}(3)\right)$, which correlate with changes in the cellular transmembrane (TM) potential. The data were analyzed using spectral unmixing methods previously described $[33,34]$ and were compared to the usual tests of the rate of survival after irradiation [12].

\section{Materials and methods}

\subsection{Bacterial Sample Preparation}

Bacterial cultures were prepared under three different conditions, as described next:

(a) Bacterial culture (MRSA USA300, strain ATCC $^{\circledR}$ BAA-1680) from the American Type Culture Collection (Manassas, VA, USA) was diluted to a concentration of $5 \times 10^{6} \mathrm{CFU} / \mathrm{mL}$, in $0.9 \%$ sterile saline, then streaked onto individual wells in 24-well microplates containing tryptic soy agar and subjected to single- or double-dose irradiation with $121 \mathrm{~J} / \mathrm{cm}^{2} 405 \mathrm{~nm}$ laser light as described below. The time interval for the double irradiation was 30 minutes, based on our previous report [17]. After treatment, the microplates were incubated for 24 hours at $37^{\circ} \mathrm{C}$. At the end of the incubation period, standardized digital images of MRSA colonies were taken from 10 $\mathrm{cm}$ above each microplate, to ensure consistency of colony magnification. Colonies were then counted, and average survival rates and standard deviations were computed and plotted as detailed previously [12, 15-17].

(b) MRSA USA300 culture described above was prepared at a concentration of $7 \times 10^{6}$ $\mathrm{CFU} / \mathrm{mL}$ with tryptic soy broth. Then, the bacterial suspension was mixed with a $1 \mathrm{mM}$ aqueous 
solution of the fluorescent dye $\mathrm{DiOC}_{2}(3)$ (Life Technologies, Grand Island, NY, USA) in dimethyl sulfoxide (DMSO) (Sigma Aldrich, St. Louis, MO, USA), with the dye solution reaching a final concentration of $18 \mu \mathrm{M}$. Aliquots of the bacterial suspension were then placed on a glass-bottom gridded dish ( $\mu$-Dish, Ibidi, Planegg, Germany) with $50 \mu \mathrm{m}$ grid size that was pre-coated with concanavalin A (Sigma Aldrich, St. Louis, MO, USA). The pre-coating followed a procedure adapted from Asakawa et al. [35] and consisted of spreading $200 \mu \mathrm{L}$ aqueous solution of concanavalin A $(0.5 \mathrm{~g} / \mathrm{L})$ and incubating the dish for 1 hour at room temperature. The excess solution was extracted from the dish using a pipette and the dish was dried overnight.

(c) MRSA USA300 culture described above was diluted with $0.9 \%$ sterile saline to a concentration of $7 \times 10^{6} \mathrm{CFU} / \mathrm{mL}$ and then placed on a gridded dish (50 $\mu \mathrm{m}$ grid size) pre-coated with concanavalin A (Sigma Aldrich, St. Louis, MO, USA) as described under protocol (b).

\subsection{Sample Irradiation by Violet/Blue Laser Light}

The setup and parameters used for irradiating the bacteria (Fig. 1, Table 1), employed a $405 \mathrm{~nm}$ original equipment manufacturer (OEM) laser assembly (Visible Diode Lasers, Cocoa, FL, USA) delivering continuous-wave (CW) light with spectral bandwidth of less than $1 \mathrm{~nm}$ and output power of $500 \mathrm{~mW}$. The slightly diverging laser beam with a cross-sectional area of $3 \mathrm{~mm}^{2}$ was expanded and then collimated to $3 \mathrm{~cm}^{2}$, which approximately matched the area of the plate. The power density measured at the sample plane was $135 \mathrm{~mW} / \mathrm{cm}^{2}$. The sample was placed on a translation stage about $25 \mathrm{~cm}$ from the collimating lens. The treatment time selected was 15 minutes, which corresponds to a fluence of $121 \mathrm{~J} / \mathrm{cm}^{2}$.

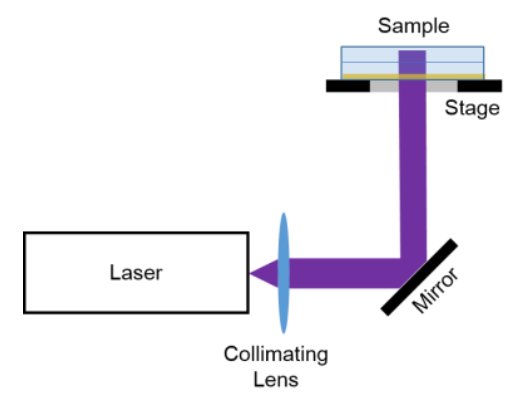

Fig. 1. Experimental setup used in bacteria irradiation with $405 \mathrm{~nm}$ laser light. The culture plates or dishes are placed on a stage whose height and position may be adjusted as necessary. The original, divergent, laser beam is collimated using a lens (focal length, $35 \mathrm{~mm}$ ) placed at a distance of $22 \mathrm{~mm}$ away from the laser aperture. The final beam diameter was $\sim 20 \mathrm{~mm}$. 
Table 1. Device information, irradiation and treatment parameters

\begin{tabular}{|c|c|c|}
\hline \multirow[t]{2}{*}{ Device } & \multicolumn{2}{|c|}{ Parameters } \\
\hline & Irradiation & Treatment \\
\hline Visible Diode Laser & Center wavelength: $405 \mathrm{~nm}$ & Beam spot size at target: $3 \mathrm{~cm}^{2}$ \\
\hline Laser model: OEM CW Laser & Spectral bandwidth: $<1 \mathrm{~nm}$ & Average irradiance at target: $135 \mathrm{~mW} / \mathrm{cm}^{2}$ \\
\hline Produced in 2013 & Continuous wave $(\mathrm{CW})$ & Exposure duration: $900 \mathrm{sec}$ \\
\hline One emitter & Total radiant power: $500 \mathrm{~mW}$ & Radiant exposure: $121 \mathrm{~J} / \mathrm{cm}^{2}$ \\
\hline Diode LASER & Aperture Diameter: $0.6 \mathrm{~cm}$ & Radiant energy: $363 \mathrm{~J}$ \\
\hline \multirow[t]{6}{*}{ Free space delivery system } & Irradiation at aperture: & Area irradiated: $3 \mathrm{~cm}^{2}$ \\
\hline & $1,770 \mathrm{~mW} / \mathrm{cm}^{2}$ & \\
\hline & Beam profile: Gaussian & $\begin{array}{l}\text { Application technique: Light was applied } \\
\text { to bacteria in a well plate using free space } \\
\text { irradiation from the laser. }\end{array}$ \\
\hline & & Number and frequency of treatments: \\
\hline & & Double irradiation, 30-min time interval. \\
\hline & & Total Radiant energy: $726 \mathrm{~J}$ \\
\hline
\end{tabular}

\subsection{Optical Setup for Fluorescence-Based Detection of Endogenous Porphyrins}

To detect fluorescence emission from the MRSA sample prepared according to protocol (c) above, we excited the sample via the same single-photon absorption process employed for eradication. Therefore, to excite the sample, we used a CW violet laser (LP405-SF30, Thorlabs, Newton, NJ) with the same wavelength $(405 \mathrm{~nm})$ as the one used for eradication. Scanning of the laser beam across the entire sample field of view was accomplished with an OptiMiS scanning module obtained from Aurora Spectral Technologies (Milwaukee, WI, USA); the scanning head was modified to permit the use of CW lasers as well as incorporate a line-scan protocol (32). The laser beam was fed through the rear port, typically used for epi-fluorescence illumination, of an inverted microscope (Axio Observer - Z1, Carl Zeiss, Oberkochen, Germany), and was reflected by a dichroic mirror (mounted in the filter cube turret) to the microscope objective (Fig. 2). The excitation light $(0.5 \mathrm{~mW})$ was focused to a line in the plane of the sample (at an irradiance of $229 \mathrm{~W} / \mathrm{cm}^{2}$ for $25 \mathrm{~ms}$ per image line) by the cylindrical mirror, scaning lens, tube lens and the $63 \mathrm{x}$ water-immersion objective $(\mathrm{NA}=1.2)$ comprising the optical excitation path. 
This irradiance resulted in a fluence of $5.7 \mathrm{~J} / \mathrm{cm}^{2}$ (and total radiant energy of a few $\mu \mathrm{J}$ ) which is much lower than that used for blue-light treatment and was therefore ignored in the results shown in Table 1.

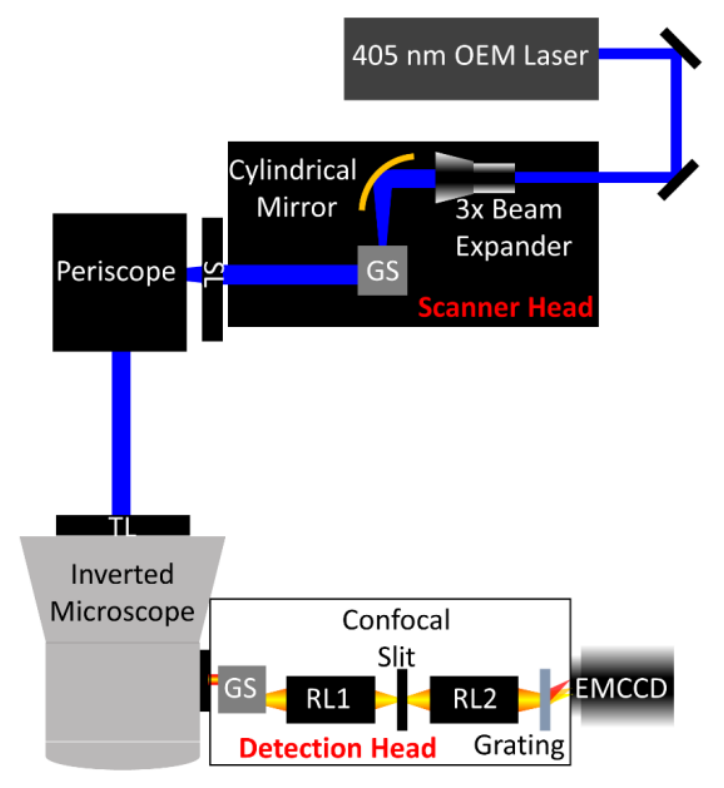

Fig. 2. Schematic diagram of the optical setup. The excitation laser is fed into the scanner head which provides the necessary optics for shaping the laser beam into a line in the sample plane, i.e., the cylindrical mirror, and a galvanometric scanner for scanning the line in the plane of the sample. A custom built periscope raises the excitation beam to the height of the back port of the inverted microscope. A pair of lenses, one serving as a scan lens (SL) and one as a tube lens (TL) are mounted directly in the scanning head and periscope, respectively. A dichroic beam splitter mounted in the filter cube turret of the inverted microscope directs the excitation beam to the microscope objective. The emission emanating from the excited sample passes back through the microscope objective, and the inverted microscope tube lens to a side port image plane. The emission is descanned by a pair of galvanometric mirrors (GS). A set of achromatic lenses (RL1), with focal lengths $75 \mathrm{~mm}$, relays the image plane of the microscope to the location of a confocal slit, and a second set of achromatic lenses (RL2), with focal lengths 75 and $150 \mathrm{~mm}$, relays the image from the location of the slit to the electron multiplying charge-coupled device (EMCCD) camera. Prior to striking the EMCCD, the light passes through a transmission grating (Grating) which spreads the emission into its spectral components along one direction of the EMCCD.

The fluorescence emission was collected with a detection head, built in house and mounted to the side port of the microscope, by relaying the fluorescent signal from the image plane of the microscope to a cooled electron-multiplying charge-coupled device (Andor iXon Ultra-DU897 back-illuminated EMCCD Andor Technologies, South Windsor, CT, USA). The fluorescence signal was actively descanned prior to striking the EMCCD detector with a pair of 
mirrors attached to a galvanometer, and mounted in the detection head enclosure (see Fig. 2). Two mirrors were mounted face-to-face onto the axle of a galvanometer scanner, which was controlled by the same program that controls the galvanometer (located in the scanning head enclosure; see Fig. 2) responsible for scanning the excitation line across the sample field of view. The emitted fluorescence was picked up by the edge of the first mirror, reflected towards the opposite edge of the second mirror, and than reflected toward the optical grating and the EMCCD camera. The active descanning galvanometer moved the two mirrors in concert with the galvanometer responsible for sweeping the excitation line through the sample field of view (located in the scanning head enclosure; see Fig. 2). In this way, the movement of the fluorescence beam emitted by the sample was compensated for by the opposite movement of the pair of mirrors in the detection head.

Actively descanning the fluorescent signal rendered the emission light beam immobile and facilitated the placement of a confocal slit in the detection path and thereby permitted the incorporation of the $\mathrm{CW}$ laser for confocal imaging. Use of a $\mathrm{CW}$ laser significantly minimizes the laser-induced photo-bleaching of the cellular fluorescence, which is known to be stronger in two- or multi-photon excitation. This was crucial for our ability to preserve and detect the dim porphyrin signal from MRSA. The fluorescence emission was spectrally resolved as previously described $(32,36,37)$. The final fluorescence photomicrographs were reconstructed for each individual emission wavelength channel (total of 300 channels; 400-700 nm spectral window) as previously described [33].

\subsection{Fluorescence-based determination of the transmembrane potential}

To detect changes in the transmembrane potential of irradiated bacteria, MRSA cells stained with the fluorescent probe $\mathrm{DiOC}_{2}(3)$, were immobilized on a gridded dish, following the protocol (b) described above, and imaged using an optical micro-spectroscope (OptiMiS TruLine, Aurora Spectral Technologies, Milwaukee, WI, USA). Two-photon excitation of the sample was achieved using a tunable mode-locked Ti:Sapphire laser (Mai Tai, Spectra Physics, Santa Clara, CA, USA), which delivered ultrashort pulses (with a bandwidth of $7 \mathrm{~nm}$ ) centered around a 880-nm wavelength, at an average power of $0.05 \mathrm{~mW} /$ pixel (i.e., an irradiance of 33 $\mathrm{W} / \mathrm{cm}^{2}$ for $25 \mathrm{~ms}$ per image line), measured after passing through the microscope objective. This resulted in a radiant exposure of $0.8 \mathrm{~J} / \mathrm{cm}^{2}$ (and total radiant energy of a few $\mu \mathrm{J}$ ), which is much lower than that used for blue-light treatment and is absorbed orders of magnitude less efficiently 
than blue light, because of the non-linear nature of the two-photon absorption effect used in this imaging assay. The laser and the OptiMiS TruLine module were attached to a Nikon Eclipse TiU inverted microscopy platform (Nikon, Melville, NY, USA). Six different fields of view were captured for each sample investigated at each time point. Acquisition of an entire bundle of spectrally resolved images (consisting of 100 images, each covering a bandwidth of $2 \mathrm{~nm}$ ) took about 10 seconds. Full description of the imaging apparatus is given elsewhere [32].

\subsection{Analysis of Fluorescence Images}

In order to separate (or unmix) the fluorescence emission from bacteria, which was either intrinsic (emitted by porphyrins, NADH, and FAD) or extrinsic (from the fluorescent dye used as a TM potential probe), we used the individual spectra of the various fluorescent species and spectral unmixing algorithm described in detail previously [33, 36, 37]. This provided fully separated fluorescence micrographs for each individual fluorescent species, the number of which was different for each type of assay (see below).

In one of the imaging assays performed in this study, which was aimed at detecting the presence of endogenous porphyrins, we needed to measure the fluorescence spectra of NADH and FAD separately. These spectra were acquired from NADH and FAD solutions using OptiMiS TruLine in a two-photon excitation configuration. NADH emission exhibited a peak at $480 \mathrm{~nm}$ while the FAD spectrum peaked at $535 \mathrm{~nm}$, which were both similar to the literature [38]. Since various types of porphyrins may exist in the bacteria [20] whose overall spectra are not fully known, the unmixing process started from the known individual NADH and FAD spectra and a Gaussian function that accounted for the unknown spectrum of porphyrin.

In a separate imaging assay, we aimed to detect changes in the fluorescence emission peak of the potential-sensitive fluorescent dye $\mathrm{DiOC}_{2}(3)$. Since the two spectra are mixed together to various degrees, depending on the exact value of the TM potential in each cell and the proximity of each fluorescent molecule to the cell membrane, we needed to unmix the fluorescence images in order to quantify the proportion of green and red color in each cell. In order to determine the elementary spectra of the green and red component, we took advantage of the fact that the emission spectrum of $\mathrm{DiOC}_{2}(3)$ shifts from green (with a peak at $515 \mathrm{~nm}$ ) to red (peaking at $580 \mathrm{~nm}$ ) as the concentration changes from low to high values. We therefore used a low concentration of $300 \mu \mathrm{M}$ to determine the spectrum of the green component and a concentration of $3 \mathrm{mM}$ to determine the spectrum of the red component. These spectra were then 
used to unmix the composite spectrum of the cells using the algorithm described above in this section.

\section{Results}

Previous studies [8, 9, 12, 14-17] have indicated that, when irradiated by blue light, MRSA loses its viability, as demonstrated by a dramatically reduced number of colonies on culture plates incubated for 24 hours at $37^{\circ} \mathrm{C}$. In order to confirm that the narrow-band violet/blue laser light $(405 \mathrm{~nm})$ used in the present work has the desired effect of inactivating these bacteria at the chosen fluence of $121 \mathrm{~J} / \mathrm{cm}^{2}$, we compared the colony-forming ability of irradiated bacteria compared to control (i.e., non-irradiated cells). As seen in Fig. 3, this was indeed the case. In addition, two equal light doses administered 30 minutes apart were more effective in de-activating the bacteria than a single dose was.
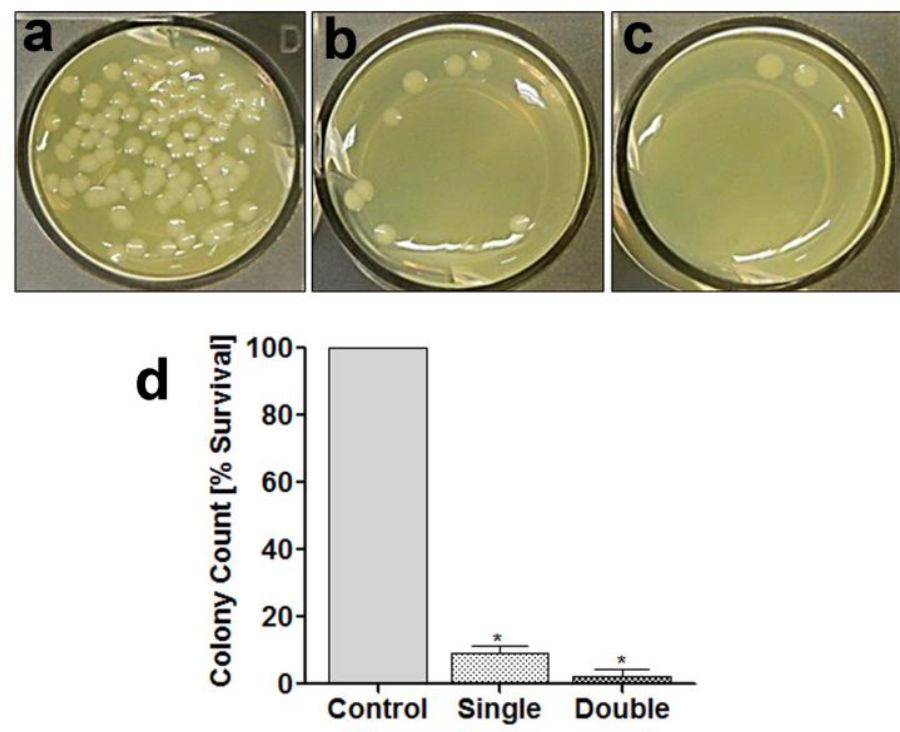

Fig. 3. Representative photographs of MRSA culture plates 24 hours after incubation at $37^{\circ} \mathrm{C}$ and survival rates. (a) Bacterial colonies of non-irradiated plate (control). Bacterial colonies of plates irradiated with $121 \mathrm{~J} / \mathrm{cm}^{2}$ of $405 \mathrm{~nm}$ laser light (b) Single irradiation, (c) Double irradiation. The interval between irradiations was 30 minutes. (d) Bar charts indicating the survival rates of MRSA after irradiation with $405 \mathrm{~nm}$ laser light, under the same experimental conditions as in the following figures; results are presented as mean $\pm \mathrm{SD}(\mathrm{n}=3)$. Asterisk indicates that changes for the treatment groups were statistically significant compared to the control.

Previous work suggested that inactivation of various bacteria, including S. aureus, occurs via optical excitation of porphyrins, which then transfer the absorbed energy to optically-inactive molecular oxygen to generate highly reactive free oxygen radicals [26]. Since that work relied on 
porphyrins produced upon induction by $\delta$-ALA while the present study does not use such induction, we wanted to test whether our MRSA samples naturally produce detectable amounts of porphyrins, which could account for the eradication of bacteria using violet/blue laser light. We have observed that many, though not all, bacteria expressed quantifiable amounts of porphyrins, as revealed by detectable fluorescence above the common auto-fluorescence background consisting of a mixture of NADH and FAD signals. Fig. 4 shows typical results obtained from bacteria that presented fluorescence emission in the spectral region where porphyrins are expected to emit [20].

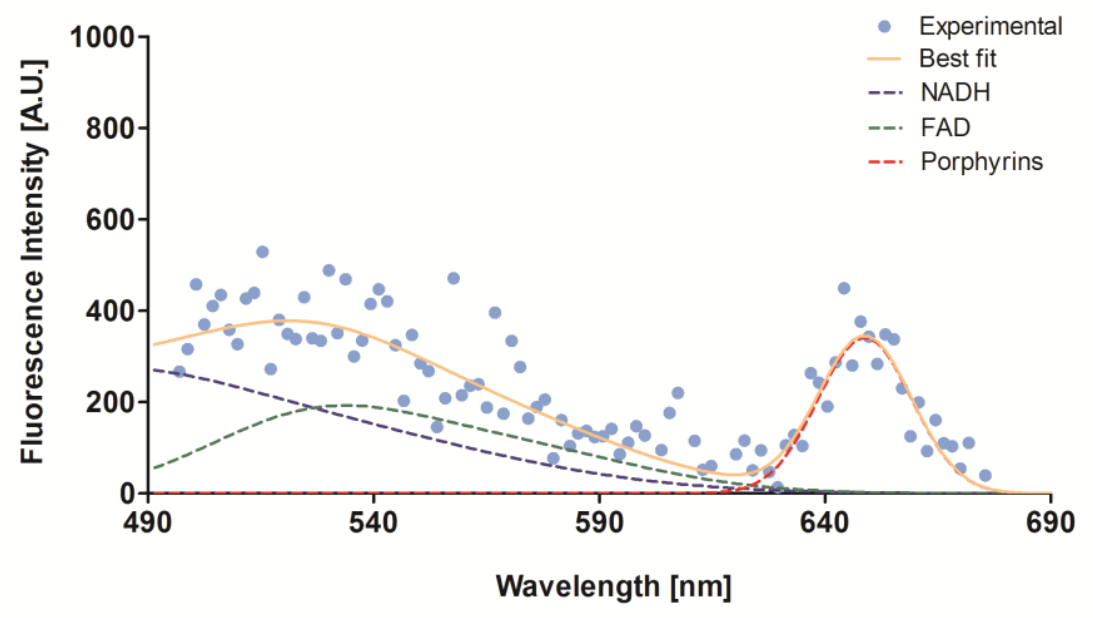

Fig. 4. Experimental emission spectrum and its theoretically unmixed components as measured using a custom-made confocal microscope with spectral resolution. The measured intensities were averaged over all the pixels corresponding to a single bacterium in an image. A leastsquares minimization algorithm (described in the methods section) indicated that the composite spectrum may be fitted to a sum of NADH, FAD and porphyrins spectra.

The observation that not all cells presented detectable amounts of porphyrins seems to indicate that MRSA produce porphyrins only during certain phases of the cell cycle and therefore are susceptible to photo-inactivation only while expressing porphyrins. This is consistent with the results presented in Fig. 3, which showed that two doses of blue light administered 30 minutes apart more effectively reduced the number of viable cells than a single dose, which may imply that 30 minutes time is long enough for more bacteria to enter the appropriate cell cycle phase and produce porphyrins. 
Having established the efficacy of our bacterial photo-inactivation treatment and that it was probably related to the presence of radical producing porphyrins, we wanted to detect possible changes in the TM potential of the cells, which is often used as an indicator of viability. To do this, the cells were stained with the fluorescent probe $\mathrm{DiOC}_{2}(3)$ (at a concentration of 18 $\mu \mathrm{M}$ ), immobilized on a gridded, glass-bottom dish, and imaged using an optical microspectroscope with two photon excitation. (We also tried a concentration of $2 \mu \mathrm{M} \mathrm{DiOC}_{2}(3)$, but this led to low signal.) The emission color of this dye is known to shift from green (with a peak at $515 \mathrm{~nm}$ ) to red (peaking at $580 \mathrm{~nm}$ ) as it is concentrated inside the bacterial cells, if the cells maintain their TM potential, and it shifts back to green if the TM potential decreases in magnitude. It is noteworthy that the concentration of dye found to be optimal in our work (18 $\mu \mathrm{M})$ was lower than that at which the dye appeared green in solution (300 $\mu \mathrm{M}$, see above). The fact that the dye in the cells emitted red fluorescence was due to its binding to cell membrane and its intracellular uptake driven by the trans-membrane potential, which lead to comparatively high concentrations in cellular compratments; hence the dye emited red fluorescence. When the TM potential decreases, the process is reversed and the intracellular concentration decrease, a process accompaniend by change of color to green.

To monitor transmembrane potential changes, the sample was irradiated, imaged 5 and 25 minutes after the first irradiation, and then irradiated with a second dose of light starting 30 minutes after the end of the first dose. Following the second irradiation step, more images were acquired sequentially at different time intervals. All spectrally resolved images were unmixed to obtain green-only and red-only images (Fig. 5).

When viewed with the optical micro-spectroscope, the viable bacteria appeared mostly red-colored, as expected (column (a) in Fig. 5), while treatment with blue laser light resulted in a clear change from red to green of the fluorescence emitted by most of the bacteria, which indicated a dramatic change in the magnitude of the TM potential (columns (b) through (e)). These visual cues were quantified by determining the average intensity for each cell in an image of green fluorescence and, similarly, for red fluorescence, computing the ratio Red/(Red+Green) for each bacterial cell and plotting the results in Fig. 5(f) as a function of time. The decreasing value of the fluorescence ratio following one and two doses of irradiation with blue laser light is apparent for all cells captured in the field of view shown in Fig. 5. 

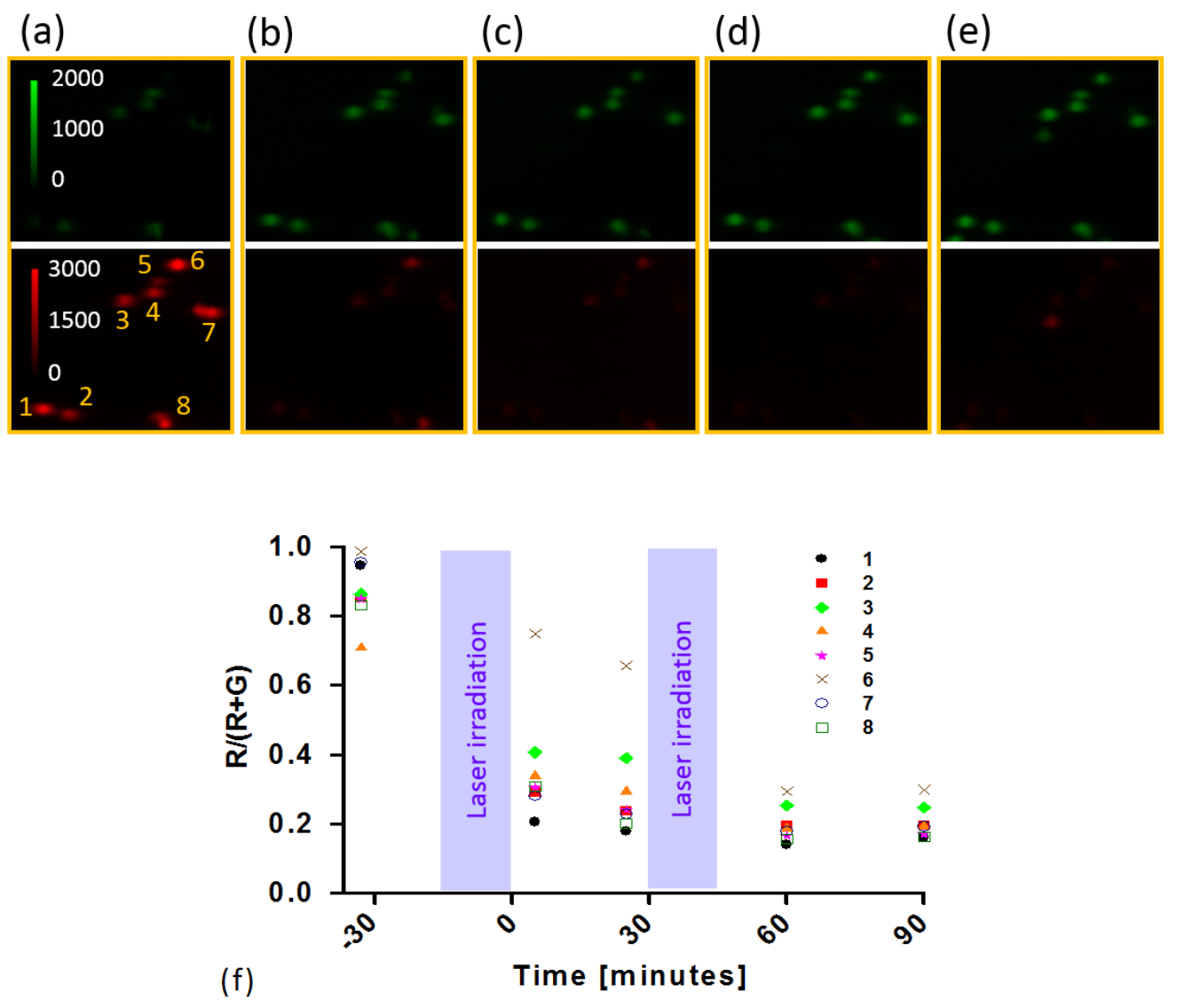

Fig. 5. Fluorescence images, acquired using an optical micro-spectroscope, of MRSA bacteria stained with $18 \mu \mathrm{M}$ of a transmembrane potential-sensitive dye $\left(\mathrm{DiOC}_{2}(3)\right)$ and subjected to the same irradiation conditions as the doubly treated sample in Figure 3. The spectrally resolved images were unmixed to obtain green-only (first row from the top) and red-only (second row) images, based on prior knowledge of the individual emission spectra (see the methods section). The spectral unmixed imaged shown were acquired before irradiation with $405 \mathrm{~nm}$ laser light (panel a), immediately (b) and 25 minutes after the first irradiation (c), respectively, and then 10 minutes (d) and 40 minutes (e) after a second irradiation with $405 \mathrm{~nm}$ laser light. The ratio $\mathrm{Red} /(\operatorname{Red}+\mathrm{Green})$ for each bacterial cell (labelled by numbers 1 to 8 in the images shown in the second row) was plotted against the time lapsed from the beginning of the experiment. The times at which $405 \mathrm{~nm}$ light doses were administered are indicated by blue rectangles.

The assay summarized in Fig. 5 was repeated for several fields of view, containing a total of 61 irradiated and 67 control (i.e., non-irradiated) cells. Individual averages were computed for each field of view, and the weighted averages were computed for all fields of view using the variance (i.e., the square of the standard deviation) for each field of view as a weighting factor. Also, standard errors were computed by adding the inverse of the SDs in quadrature. The results, shown in Fig. 6, correlated well with the cellular viability as determined by the ability of 
individual bacteria to form colonies (Fig. 3). In addition, the marked contrast between the $\mathrm{Red} /(\mathrm{Red}+\mathrm{Green})$ ratios in the presence or absence of blue-light irradiation indicates that DMSO, which was used as a solvent for the dye and is known to adversely affect mitochondrial function after prolonged exposure, has no significant effect on cell viability, at least when compared to the effect of blue light irradiation.

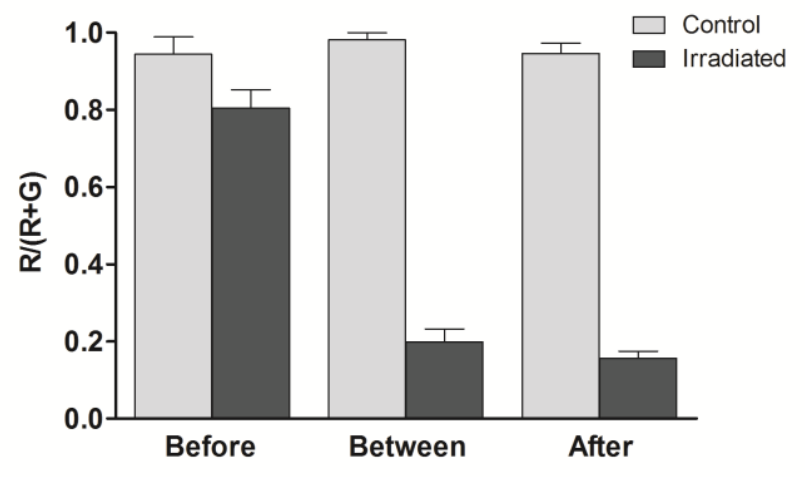

Fig. 6. Change in the Red/(Red+Green) ratio averaged over 61 and 67 cells for the irradiated (dark grey bars) and control (light grey bars) samples, respectively, before, in between, and after two irradiations with $405 \mathrm{~nm}$ laser light. The irradiations lasted 15 minutes each and were 30 minutes apart, as measured from the end of the first to the beginning of the second dose.

This correlation suggests that the loss of viability of bacterial cells exposed to blue laser light is due to a mechanism that also induces marked changes in the TM potential, such as disabling ion pumps or alterations in membrane structural integrity (at molecular level) that make the membrane more permeable to ions. In either case, the cell would become unable to maintain its ion concentration asymmetry between the cytoplasm and the extracellular medium, which causes a drop in the chemical potential differences and a corresponding drop in the difference between the electrical potentials across the plasma membrane. Previous observation that S. aureus undergo significant decrease in their $\mathrm{Na}^{+}$and $\mathrm{K}^{+}$contents [20] after exposure to light in the 407-420 $\mathrm{nm}$ range lends additional support to this hypothesis.

\section{Discussion}

Generally, the phenotypic alterations in resistant $S$. aureus can be grouped into (1) changes in cell wall structure and turnover; (2) changes in membrane composition, structure and potential; (3) modification in sensitivity to depolarization, autolysis and permeabilization. Some antibiotic therapies have explored these changes as potential targets for bacterial suppression [39]. Of great physiological importance to any biological cell is the membrane potential [40], 
which is a component of the proton motive force involved in ATP generation, implicated in bacterial autolysis, glucose transport, chemotaxis, and survival of bacteria at low $\mathrm{pH}$ [41]. Therefore, depolarization of the cell membrane results in malfunction of these physiological processes in bacteria and ultimately leads to death.

The membrane potential of metabolically active bacteria is typically on the order of -150 $\mathrm{mV}$, with the cell interior being electrically negative relative to the extracellular side of the membrane [41]. Evidence is provided here that blue light causes a depolarization of MRSA membrane as depicted by the shift in the emission spectrum, of $\operatorname{DiOC}_{2}(3)$, a dye utilized for analysis of transmembrane potential. A reduction in the red $\mathrm{DiOC}_{2}(3)$ fluorescence associated with viable bacteria (and increase in green fluorescence) was indicative of a reduction in the potential of the electrically negative interior. Previous studies [42] showed that an increase in the magnitude if the membrane potential correlated with changes in the equilibrium between free dye and dye bound to the plasma membrane and led to an increase in dye intracellular concentration. These resulted in a color change from green outside of the cell to red as it was trsported inside the cell. Reduction of the TM potential reversed the color change inside the cell from red to green. In our study, the reduction in the transmembrane potential as evidenced by the change in color of the voltage sensitive dye correlated with rapid percentage suppression of MRSA growth, providing direct evidence that the effect of blue light irradiation is immediate and alters the cellular viability rather than, for example, inducing mutations that result in reduced long-term viability of an entire population of bacteria. Experimental data in this study also provided evidence for the presence of porphyrins in MRSA, which provide the necessary link between irradiation with blue/violet light and the decrease in the transmembrane potential, most likely, via reactive oxygen species attack of the membrane. In addition, the fact that all cells did not always express detectable amounts of porphyrins indicated that production of these photosensitizers depends on the cell cycle phase and suggested that two or more doses of light could be more effective than a single dose of equal fluence. This was indeed confirmed experimentally by assessing the rate of survival of bacterial colonies.

\section{Conclusion}

The results presented in this paper provide very useful clues regarding the mechanisms underlying blue light-suppression of MRSA activity, which are crucial to the development of treatment modalities for infection. Our findings suggest that the early antimicrobial activity of 
blue light results from alteration of membrane integrity with a consequent decrease in membrane polarization and alteration of vital cellular functions. By contrast, the standard incubation procedure could only suggest a limited ability of the cells to multiply, as indicated by reduction number in the number of colonies of light-irradiated bacteria after 24-hour incubation. The observation of an early antimicrobial activity of light is very encouraging, as it suggests that treatment does not necessarily have to be administered over a long period of time.

\section{Acknowledgments}

This work was partly supported by grants from the National Science Foundation's Major Research Instrumentation Program (grant PHY-1126386 awarded to V.R.) and the Bradley Foundation/UWM Research Foundation (Catalyst grant MIL 106970 awarded to C.E. and V.R.).

\section{References}

[1] H. H. Liu, Antibiotic resistance in bacteria. A current and future problem, Adv. Exp. Med. Biol. 455 (1999) 387-396.

[2] R. S. Howell-Jones, M. J. Wilson, K. E. Hill, A. J. Howard, P. E. Price, D. W. Thomas, A review of the microbiology, antibiotic usage and resistance in chronic skin wounds, J. Antimicrob. Chemother. 55 (2005) 143-149.

[3] J. Graham, Deaths from drug-resistant bacteria top those from AIDS. Chicago Tribune (2007).

[4] P. Muller, D. G. Alber, L. Turnbull, R. C. Schlothauer, D. A. Carter, C. B. Whitchurch, E. J. Harry, Synergism between Medihoney and rifampicin against methicillin-resistant Staphylococcus aureus (MRSA), PLoS One 8 (2013) e57679.

[5] C. C. Otto, S. E. Haydel, Exchangeable ions are responsible for the in vitro antibacterial properties of natural clay mixtures, PLoS One 8 (2013) e64068. 
[6] I. Tsuneyoshi, W. A. Boyle, 3rd, Y. Kanmura, T. Fujimoto, Hyperbaric hyperoxia suppresses growth of Staphylococcus aureus, including methicillin-resistant strains, J. Anesth. 15 (2001) 2932.

[7] V. Turhan, S. Sacar, G. Uzun, M. Sacar, S. Yildiz, N. Ceran, R. Gorur, O. Oncul, Hyperbaric oxygen as adjunctive therapy in experimental mediastinitis, J. Surg. Res. 155 (2009) 111-115.

[8] C. S. Enwemeka, D. Williams, S. Hollosi, D. Yens, S. K. Enwemeka, Visible 405 nm SLD light photo-destroys methicillin-resistant Staphylococcus aureus (MRSA) in vitro, Lasers Surg. Med. 40 (2008) 734-737.

[9] C.S. Enwemeka, D. Williams, S. Hollosi, S.K. Enwemeka, S. Hollosi, D. Yens, Blue 470-nm light kills methicillin-resistant Staphylococcus aureus (MRSA) in vitro, Photomed. Laser Surg. 27 (2009) 221-226.

[10] A. Lipovsky, Y. Nitzan, H. Friedmann, R. Lubart, Sensitivity of Staphylococcus aureus strains to broadband visible light, Photochem Photobiol 85 (2009) 255-260.

[11] T. Dai, A. Gupta, Y. Y. Huang, M. E. Sherwood, C. K. Murray, M. S. Vrahas, T. Kielian, M. R. Hamblin, Blue light eliminates community-acquired methicillin-resistant Staphylococcus aureus in infected mouse skin abrasions, Photomed. Laser Surg. 31 (2013) 531-538.

[12] V.V. Bumah, D.S. Masson-Meyers, S.E. Cashin, C.S. Enwemeka, Wavelength and bacterial density influence the bactericidal effect of blue light on methicillin-resistant Staphylococcus aureus (MRSA), Photomed. Laser Surg. 31 (2013) 547-553.

[13] M. Maclean, K. McKenzie, J. G. Anderson, G. Gettinby, S. J. MacGregor, 405 nm light technology for the inactivation of pathogens and its potential role for environmental disinfection and infection control, J. Hosp. Infect. 88 (2014) 1-11.

[14] V.V. Bumah, D.S. Masson-Meyers, S.E. Cashin, C.S. Enwemeka, Optimization of the antimicrobial effect of blue light on methicillin-resistant Staphylococcus aureus (MRSA) in vitro, Lasers Surg. Med. 47 (2015) 266-272.

[15] V.V. Bumah, D.S. Masson-Meyers, B.J. Quirk, E. Buchmann, H.T. Whelan, C.S. Enwemeka, The bactericidal effect of $470 \mathrm{~nm}$ light and hyperbaric oxygen on methicillinresistant Staphylococcus aureus (MRSA), Lasers Med. Sci. 30 (2015) 1153-1159.

[16] V. V. Bumah, D. S., Masson-Meyers, C. S. Enwemeka, Blue $470 \mathrm{~nm}$ light suppresses the growth of Salmonella enterica and methicillin-resistant Staphylococcus aureus (MRSA) in vitro, Lasers Surg. Med. 47 (2015) 595-601. 
[17] D.S. Masson-Meyers, V.V. Bumah, G. Biener, V. Raicu, C.S. Enwemeka, The relative antimicrobial effect of blue $405 \mathrm{~nm}$ LED and blue $405 \mathrm{~nm}$ laser on methicillin-resistant Staphylococcus aureus in vitro, Lasers Med. Sci. 30 (2015) 2265-2271.

[18] P. Papageorgiou, A. Katsambas, A. Chu, Phototherapy with blue (415 nm) and red (660 nm) light in the treatment of acne vulgaris, Br. J. Dermatol. 142 (2000) 973-978.

[19] H. Ashkenazi, Z. Malik, Y. Harth, Y. Nitzan, Eradication of Propionibacterium acnes by its endogenic porphyrins after illumination with high intensity blue light, FEMS Immunol. Med. Microbiol. 35 (2003) 17-24.

[20] Y. Nitzan, M. Salmon-Divon, E. Shporen, Z. Malik, ALA induced photodynamic effects on Gram positive and negative bacteria, Photochem. Photobiol. Sci. 3 (2004), 430-435.

[21] K. Morimoto, T. Ozawa, K. Awazu, N. Ito, N. Honda, S. Matsumoto, D. Tsuruta, Photodynamic therapy using systemic administration of 5-aminolevulinic acid and a 410-nm wavelength light-emitting diode for methicillin-resistant Staphylococcus aureus infected ulcers in mice, PLoS One 9 (2014) e105173.

[22] M. Maclean, S. J. MacGregor, J. G. Anderson, G. Woolsey, "Inactivation of bacterial pathogens following exposure to light from a 405-nanometer light-emitting diode array," Appl Environ Microbiol 75, 1932-1937 (2009).

[23] A. Lipovsky, Y. Nitzan, A. Gedanken, R. Lubart, Visible light-induced killing of bacteria as a function of wavelength: implication for wound healing, Lasers Surg. Med. 42 (2010) 467-472.

[24] C. J. Wasson, J. L. Zourelias, N. A. Aardsma, J. T. Eells, M. T. Ganger, J. M. Schober, T. A. Skwor, Inhibitory effects of $405 \mathrm{~nm}$ irradiation on Chlamydia trachomatis growth and characterization of the ensuing inflammatory response in HeLa cells, BMC Microbiol. 12 (2012) 176.

[25] J. R. Lakowicz, Principles of Fluorescence Spectroscopy, Springer, New York, 2006 DOI: 10.1007/978-0-387-46312-4.

[26] H. Yang, H. Inokuchi, J. Adler, Phototaxis away from blue light by an Escherichia coli mutant accumulating protoporphyrin IX, Proc. Natl. Acad. Sci. USA 92 (1995) 7332-7336.

[27] B. Grimm, Regulatory mechanisms of eukaryotic tetrapyrrole biosynthesis, in: K. M. Guilard, K. M. Kadish, R. Smith (Eds.), The Porphyrin Handbook - The Iron and Cobalt Pigments: Biosynthesis, Structure and Degradation, Academic Press, Amsterdam 2003, pp. 1-32. [28] T. Kanazawa, S. Ren, M. Maekawa, K. Hasegawa, F. Arisaka, M. Hyodo, Y. Hayakawa, H. Ohta, S. Masuda, Biochemical and physiological characterization of a BLUF protein-EAL 
protein complex involved in blue light-dependent degradation of cyclic diguanylate in the purple bacterium Rhodopseudomonas palustris, Biochemistry 49 (2010) 10647-10655.

[29] Y. Tang, Z. Cao, E. Livoti, U. Krauss, K. E. Jaeger, W. Gartner, A. Losi, Interdomain signaling in the blue-light sensing and GTP-binding protein YtvA: a mutagenesis study uncovering the importance of specific protein sites," Photochem. Photobiol. Sci. 9 (2010) 47-56. [30] M. A. v. d. Horst, T. P. Stalcup, S. Kaledhonkar, M. Kumauchi, M. Hara, A. Xie, K. J. Hellingwerf, W. D. Hoff, Locked chromophore analogs reveal that photoactive yellow protein regulates biofilm formation in the deep sea bacterium Idiomarina loihiensis, J. Am. Chem. Soc. 131 (2009) 17443-17451.

[31] N. C. Rockwell, S. S. Martin, K. Feoktistova, J. C. Lagarias, Diverse two-cysteine photocycles in phytochromes and cyanobacteriochromes, Proc. Natl. Acad. Sci. USA 108 (2011), 11854-11859.

[32] G. Biener, M. R. Stoneman, G. Acbas, J. D. Holz, M. Orlova, L. Komarova, S. Kuchin, V. Raicu, Development and experimental testing of an optical micro-spectroscopic technique incorporating true line-scan excitation, Int J Mol Sci 15 (2014), 261-276.

[33] V. Raicu, M. R. Stoneman, R. Fung, M. Melnichuk, D. B. Jansma, L. F. Pisterzi, S. Rath, M. Fox, J. W. Wells, D. K. Saldin, Determination of supramolecular structure and spatial distribution of protein complexes in living cells, Nat. Photon. 3 (2009), 107-113.

[34] V. Raicu, Deo R. Singh, FRET Spectrometry: a new tool for the determination of protein quaternary structure in living cells, Biophys. J. 105 (2013), 1937-1945.

[35] H. Asakawa, Y. Hiraoka, T. Haraguchi, Live CLEM Imaging: an application for yeast cells, in: A. Mendez-Vilas (Ed.), Current Microscopy Contributions to Advances in Science and Technology. Microscopy Book Series number 5, Formatex Research Center, Badajoz, Spain 2012, pp. 478-485.

[36] M. R. Stoneman, J. D. Paprocki, G. Biener, K. Yokoi, A. Shevade, S. Kuchin, V. Raicu, Quaternary structure of the yeast pheromone receptor Ste2 in living cells, Biochim. Biophys. http://dx.doi.org/10.1016/j.bbamem.2016.12.008.

[37] D.R. Singh, M. Mohammad, S. Patowary, M.R. Stoneman, J. Oliver, L. Movileanu, V. Raicu, Determination of the quaternary structure of a bacterial ATP-binding cassette (ABC) transporter in living cells, Integrative Biol., 5 (2013) 312-323.

[38] S. Huang, A. A. Heikal, W. W. Webb, Two-photon fluorescence spectroscopy and microscopy of NAD(P)H and flavoprotein, Biophys. J. 82 (2002) 2811-2825. 
[39] K. Nadrah, F. Strle, Antibiotic combinations with daptomycin for treatment of Staphylococcus aureus infections, Chemother. Res. Pract. 2011 (2011) 619321.

[40] V. Raicu, and A. Popescu, Integrated Molecular and Cellular Biophysics, Springer, Netherlands, 2008 DOI: 10.1007/978-1-4020-8268-9.

[41] D. Novo, N. G. Perlmutter, R. H. Hunt, H. M. Shapiro, Accurate flow cytometric membrane potential measurement in bacteria using diethyloxacarbocyanine and a ratiometric technique, Cytometry 35 (1999) 55-63.

[42] T. K. Das, N. Periasamy, G. Krishnamoorthy, Mechanism of response of potential-sensitive dyes studied by time-resolved fluorescence, Biophys. J. 64 (1993), 1122-1132. 


\section{Figure captions}

Fig. 1. Experimental setup used in bacteria irradiation with $405 \mathrm{~nm}$ laser light. The culture plates or dishes are placed on a stage whose height and position may be adjusted as necessary. The original, divergent, laser beam is collimated using a lens (focal length, $35 \mathrm{~mm}$ ) placed at a distance of $22 \mathrm{~mm}$ away from the laser aperture. The final beam diameter was $15 \mathrm{~mm}$.

Fig. 2. Schematic diagram of the optical setup. The excitation laser is fed into the scanner head which provides the necessary optics for shaping the laser beam into a line in the sample plane, i.e. the cylindrical mirror, and a galvanometric scanner for scanning the line in the plane of the sample. A custom built periscope raises the excitation beam to the height of the back port of the inverted microscope. A pair of lenses, one serving as a scan lens (SL) and one as a tube lens (TL) are mounted directly in the scanning head and periscope, respectively. A dichroic beam splitter mounted in the filter cube turret of the inverted microscope directs the excitation beam to the microscope objective. The emission emanating from the excited sample passes back through the objective, directional back scatter detector (DBS), and the inverted microscope tube lens to a side port image plane. The emission is descanned by a pair of galvanometric mirrors (GS). A set of lenses (RL1), with focal lengths $75 \mathrm{~mm}$, relays the image plane of the microscope to the location of a confocal slit, and a second set of lenses (RL2), with focal lengths 75 and $150 \mathrm{~mm}$, relays the image from the location of the slit to the electron multiplying charge-coupled device (EMCCD) camera. Prior to striking the EMCCD, the light passes through a transmission grating (Grating) which spreads the emission into its spectral components along one direction of the EMCCD.

Fig. 3. Representative photographs of MRSA culture plates 24 hours after incubation at $37^{\circ} \mathrm{C}$ and survival rates. (a) Bacterial colonies of non-irradiated plate (control). Bacterial colonies of plates irradiated with $121 \mathrm{~J} / \mathrm{cm}^{2}$ of $405 \mathrm{~nm}$ laser light (b) Single irradiation, (c) Double irradiation. The interval between irradiations was 30 minutes. (d) Bar charts indicating the survival rates of MRSA after irradiation with $405 \mathrm{~nm}$ laser light, under the same experimental conditions as in the following figures; results are presented as mean $\pm \mathrm{SD}(\mathrm{n}=3)$. Asterisk indicates that changes for the treatment groups were statistically significant compared to the control. 
Fig. 4. Experimental emission spectrum and its theoretically unmixed components as measured using a custom-made confocal microscope with spectral resolution. The measured intensities were averaged over all the pixels corresponding to a single bacterium in an image. A leastsquares minimization algorithm (described in the methods section) indicated that the composite spectrum may be fitted to a sum of NADH, FAD and porphyrins spectra.

Fig. 5. Fluorescence images, acquired using an optical micro-spectroscope, of MRSA bacteria stained with $18 \mu \mathrm{M}$ of a transmembrane potential-sensitive dye $\left(\mathrm{DiOC}_{2}(3)\right)$ and subjected to the same irradiation conditions as the doubly treated sample in Figure 3. The spectrally resolved images were unmixed to obtain green-only (first row from the top) and red-only (second row) images, based on prior knowledge of the individual emission spectra (see the methods section). The spectral unmixed images shown were acquired before irradiation with $405 \mathrm{~nm}$ laser light (panel a), immediately (b) and 25 minutes after the first irradiation (c), respectively, and then 10 minutes (d) and 40 minutes (e) after a second irradiation with $405 \mathrm{~nm}$ laser light. The ratio Red/(Red+Green) for each bacterial cell (labelled by numbers 1 to 8 in the images shown in the second row) was plotted against the time lapsed from the beginning of the experiment. The times at which $405 \mathrm{~nm}$ light doses were administered are indicated by blue rectangles.

Fig. 6. Change in the $\operatorname{Red} /(\operatorname{Red}+$ Green $)$ ratio averaged over 61 and 67 cells for the irradiated (dark grey bars) and control (light grey bars) samples, respectively, before, in between, and after two irradiations with $405 \mathrm{~nm}$ laser light. The irradiations lasted 15 minutes each and were 30 minutes apart, as measured from the end of the first to the beginning of the second dose.

\section{Table Caption}

Table 1. Device information, irradiation and treatment parameters. 